\title{
EFECTO DE DOS SISTEMAS DE PASTOREO EN LA PRODUCCIÓN DE MATERIA SECA Y LA COMPOSICIÓN FLORÍSTICA DE UN PASTIZAL DE Andropogon lateralis Nees.
}

\author{
Hack, Claudina M.' ; Ciotti, Elsa M. ${ }^{2}$; Castelán, María E. ${ }^{1}$; Porta, Miriam'; Tomei, Carlos E. ${ }^{1}$ \\ ${ }^{1}$ Instituto Agrotécnico "Pedro M. Fuentes Godo". FCA-UNNE. Av. Las Heras 727 (3500) Resistencia-Chaco. \\ ${ }^{2}$ Facultad de Ciencias Agrarias- UNNE. \\ e-mail: claudinahack@yahoo.com.ar
}

\section{RESUMEN}

El objetivo del trabajo fue determinar la composición florística y la producción de materia seca de un pastizal de Andropogon lateralis bajo dos sistemas de pastoreo, en la región occidental de Corrientes (Argentina). Los tratamientos fueron: pastoreo continuo (T0) y pastoreo rotativo intensivo (T1). A T0 se asignó una superficie de 120 ha y a T1 una superficie de 0,25 ha. Durante la mayor parte del período de evaluación, los animales permanecieron en T0 e ingresaron a pastorear $\mathrm{T} 1 \mathrm{cada}$ vez que las plantas de menor porte tenían una altura mínima de $10 \mathrm{~cm}$ y se retiraron del potrero cuando dichas plantas alcanzaron $2,5 \mathrm{~cm}$ aproximadamente, permaneciendo entre 12 y 24 hs. Se evaluó composición florística por medio de censos y materia seca acumulada (MSA) por cortes. Los muestreos se realizaron antes del ingreso de los animales a T1, el tiempo entre cortes o pastoreos varió según el desarrollo de las plantas. Se separaron y pesaron los siguientes componentes: a) graminiforme (Gramíneas y Ciperáceas), b) Leguminosas, c) otras familias y d) material vegetal muerto. Los resultados fueron analizados con varianza y las medias se compararon con el test de Tukey $(\mathrm{p}<0,05)$. Se identificaron 31 especies, principalmente de ciclo estival. 58\% corresponde a Gramíneas, $22 \%$ a Leguminosas, $10 \%$ a Ciperáceas y el $10 \%$ restante a otras familias. En T1 se observó una mayor diversidad en la flora y aumentó la frecuencia de especies invernoprimaverales. Las gramíneas cubrieron entre el 65 y el $95 \%$, ciperáceas del 1 al $6 \%$ con un máximo de $30 \%$, leguminosas: del 1 al $13 \%$ y otras familias hasta el 7\%. La "paja colorada" (A. lateralis), fue la especie con mayor frecuencia y cobertura en el tapiz. Las Leguminosas y las demás familias de latifoliadas tuvieron una baja contribución a la producción del pastizal. El principal aporte estuvo dado por graminiformes y material muerto. El componente graminiforme tuvo una importante fluctuación estacional, registrándose el máximo en el verano y el mínimo en el invierno. T1 fue significativamente superior y prácticamente duplicó a T0 en el invierno. Alternar períodos de ocupación y descanso permite a las plan- tas recomponer sus reservas y mejorar su capacidad de rebrote, especialmente en períodos desfavorables.

Palabras clave: materia seca acumulada, composición florística, pastoreo continuo, pastoreo rotativo.

\section{ABSTRACT}

The aim of this work was to determine the floristic composition and dry matter yield of an Andropogon lateralis grassland under two pasturing systems, in the Occidental zone of Corrientes (Argentina).Treatments were: continuous (T0) and intensive (T1) grazing. T0 was carried on 120 ha and $\mathrm{T} 1$ on 0,25 ha. Animals stayed on T0 during most of the evaluation period and after each sampling, entered Tleach time plantas had $10 \mathrm{~cm}$ height and took off at $2,5 \mathrm{~cm}$.

They stayed for 12 or 24 hours depending upon forage availability. Botanic composition was studied through census and accumulated dry matter yield (DMY) through cuttings. Interval between cuttings was variable according to plant growth. Harvested material was sorted and weighed as: a) Graminaceae and Cyperaceae, b) Leguminosae, c) other families and d) plant dead material. Results were tested with variance and means were compared with Tukey test $(\mathrm{p}<0.05) .31 \mathrm{spp}$ were identified, mostly from warm season. $58 \%$ were grasses, $22 \%$ legumes; $10 \%$ Ciperaceas and $10 \%$ other families. T1 showed more plant diversity and increased frequency of winter-spring growth. Grasses covered between $65 \%$ to $95 \%$, ciperaceas $1 \%$ to $6 \%$, legumes from 1 to $13 \%$ and other families $7 \%$. A. lateralis was the species with higher frequency and percentage of cover. Legumes and other families had low contribution to grassland yield. Mean group was graminiformes and dead material. Grasses had seasonal variation, being maximum during summer and minimum in winter. T1 differed statistically from T0 during winter, being up to double. The alternation of graze and rest periods allows plant reserves to recover and improve regrowth ability, especially during unfavorable periods.

Key Words: accumulated dry matter, botanical composition, continuous or intensive grazing. 


\section{INTRODUCCIÓN}

La principal actividad agropecuaria en la Provincia de Corrientes (Argentina) es la ganadería. La misma se desarrolla fundamentalmente en campos naturales, que ocupan un $95 \%$ del área dedicada a la actividad (Royo Pallarés, 2000). La composición florística de los mismos es muy variada, con predominio de gramíneas megatérmicas. El aporte de las gramineas al rendimiento total de la materia seca varía entre el 70 y el $80 \%$. Las leguminosas tienen una contribución, que oscila entre el 3 y el $8 \%$ del total en los campos altos y es prácticamente nula en los campos bajos (Fernández, Benítez, Royo Pallarés y Pizzio, 1993; Royo Pallarés, 2000).

La producción de forraje en la zona está fuertemente influida por las variables climáticas. La mayoría de las especies vegetales que integran el tapiz son de ciclo primavero-estival, y como consecuencia la curva de crecimiento presenta marcadas variaciones estacionales que afectan directamente a la producción ganadera (Pizzio y Royo Pallarés, 1994).

El manejo del pastoreo es un factor crítico en el mantenimiento de los pastizales. En la región NEA, el sistema de pastoreo predominante es el continuo, caracterizado por tener una alta frecuencia de defoliación y una intensidad de pastoreo dependiente de la carga animal (Pizzio, 1995). Con este sistema de pastoreo, generalmente no se considera la estacionalidad productiva de las especies nativas. Tal manejo lleva a un sobrepastoreo en el período de baja productividad y a un subpastoreo en el período de alta productividad (Damè, et al. 1999). Existe una alta selectividad de las especies más palatables, que como consecuencia se deterioran y disminuyen su aporte, favoreciendo el avance y el establecimiento de otras especies de menor calidad. Con este sistema se produce una degradación paulatina del suelo y la vegetación, con la consiguiente pérdida de productividad (Bissio, 1984; Fuhlendorf et al. 2003).

En el pastoreo rotativo intensivo, la permanencia de los animales en el potrero tiene duración limita- da. Para una correcta utilización de dicho sistema, es necesario respetar el tiempo de rebrote de los pastos, regulando tanto la carga como la frecuencia de pastoreo y aumentando la eficiencia en la utilización de los pastizales (Tomei, 1995).

El objetivo de este trabajo fue determinar la composición florística y la producción de materia seca de un pastizal de Andropogon lateralis bajo dos sistemas de pastoreo.

\section{MATERIALES Y METODOS}

El trabajo se realizó en un establecimiento del Departamento Lavalle, ubicado en la Región Occidental de la provincia de Corrientes (Argentina), a $29^{\circ} 05^{\circ}$ lat $\mathrm{S}$ y $58^{\circ} 40^{\prime}$ long $\mathrm{O}$. El mismo se encuentra en el área de Lomadas Arenosas, Planicies y Depresiones (Carnevali, 1994). El suelo es un Psamacuent Acuico, serie Chavarría, con un tapiz vegetal dominado por Andropogon lateralis n.v: paja colorada (Capurro y Escobar, 1990).

El clima es subtropical sin estación seca, con veranos calurosos e inviernos benignos. Las temperaturas medias mensuales registradas durante el período de evaluación, comprendido entre septiembre de 2001 y marzo de 2003, fueron similares al promedio de los últimos 40 años. Las precipitaciones en cambio fueron muy variables. En algunos meses se registraron valores muy altos, cercanos a los $500 \mathrm{~mm}$ (Fig.1). En la mayoría de los meses el balance hídrico fue positivo, con excedentes en marzo, abril, julio y diciembre de 2002 y marzo de 2003. El déficit más importante se produjo durante el verano del primer año.

Los tratamientos fueron: pastoreo continuo (T0) y pastoreo rotativo intensivo (T1). A T0 se asignó una superficie de 120 ha y a T1 una superficie de 0,25 ha. El perímetro de T0 se delimitó con alambrado convencional y el de $\mathrm{T} 1$ con alambrado eléctrico. $\mathrm{La}$ superficie de T1 se estableció de manera que, con el mismo lote de animales utilizado para $\mathrm{T} 0$, se consumiera el forraje disponible en un breve período de tiempo. Se usaron 80 novillos, que representan una

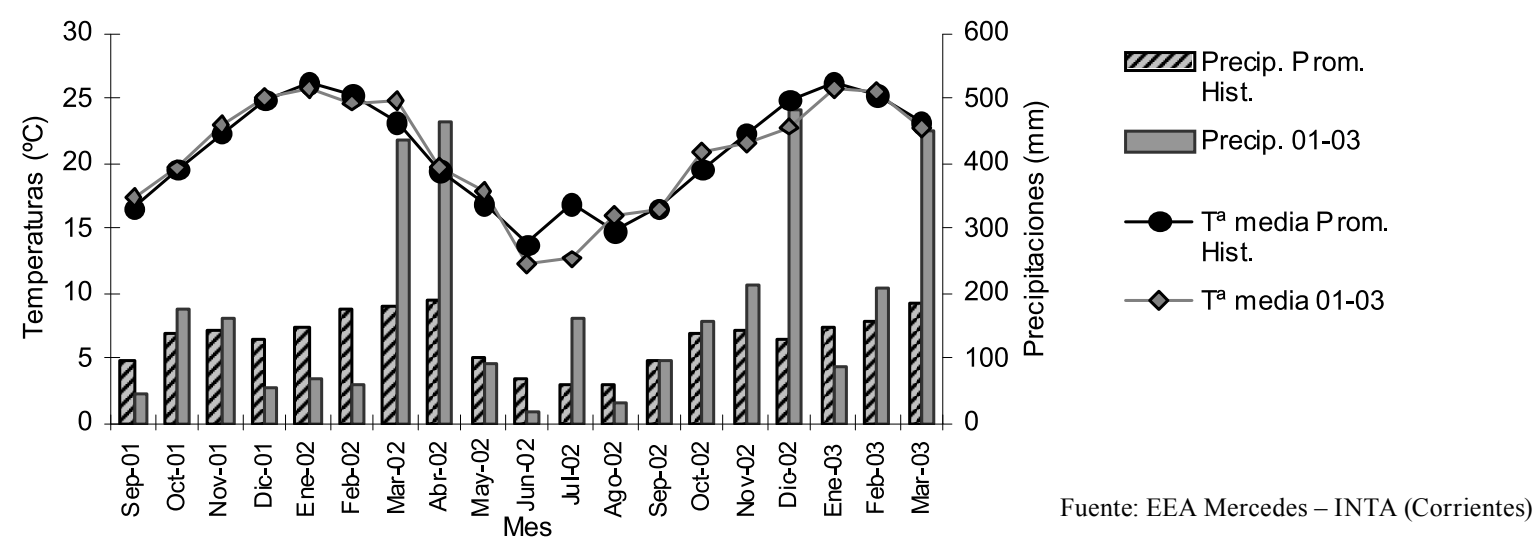

Figura 1: Temperaturas medias y precipitaciones registradas durante el período de evaluación 
carga promedio de 0,6 EV.ha-1. La mayor parte del período de evaluación los animales permanecieron en T0. Ingresaron a pastorear T1 cada vez que las plantas de menor porte tenían una altura mínima de $10 \mathrm{~cm}$ y se retiraron del potrero cuando dichas plantas alcanzaron 2,5 cm aproximadamente. En consecuencia, el tiempo de permanencia en $\mathrm{T} 1$ fue de 12 a $24 \mathrm{hs}$. Luego de ese período volvieron al potrero T0, quedando en descanso $\mathrm{T} 1$ a fin de permitir la recuperación del pastizal.

Para las evaluaciones en ambos tratamientos se establecieron dos transectas fijas en sectores donde las condiciones físico-químicas del suelo y la composición botánica inicial eran similares. Los puntos de muestreo se ubicaron sistemáticamente sobre dichas transectas y se identificaron con estacas permanentes.

Los factores analizados fueron: composición florística y materia seca acumulada (MSA). Para el primero se efectuaron tres censos: al inicio, a los siete y a los 16 meses empleando la metodología de análisis fitosociológico de Roig (1977). Se determinaron las especies del tapiz, la frecuencia de las mismas y la cobertura relativa por familia.

Para medir la acumulación de forraje se realizaron cortes. La unidad de muestreo fue de $0,25 \mathrm{~m}^{2} \mathrm{y}$ se tomaron 16 muestras por tratamiento en cada fecha de corte, alrededor de los puntos de muestreo ya descriptos. En T0 se emplearon jaulas de exclusión móviles de $1 \mathrm{~m}^{2}$ de superficie para evitar el consumo de los animales y determinar la MSA entre dos cortes. Luego de cada muestreo, las jaulas cambiaron de ubicación para evitar el efecto de los cortes anteriores. De igual manera, en T1 no se efectuaron dos cortes consecutivos sobre el mismo lugar. Los muestreos se realizaron antes del ingreso de los animales a T1. Como consecuencia, el tiempo entre cortes o pastoreos varió entre 36 y 87 días según el desarrollo de las plantas, resultando 10 fechas de corte. Se efectuaron dos cortes estivales y dos cortes primaverales en cada año, un corte en otoño y uno en invierno el primer año. El material cosechado se secó en estufa de tiro forzado a $60{ }^{\circ} \mathrm{C}$ hasta peso constante. En cada muestra se separaron y pesaron los siguientes componentes: a) graminiforme (Gramíneas y Ciperáceas), b) Leguminosas, c) otras familias y d) material vegetal muerto.

Con los datos obtenidos se calculó la acumulación de MS ( $\left.\mathrm{kg} \cdot \mathrm{ha}^{-1}\right)$ estacional y se compararon las medias de ambos tratamientos. Los resultados se sometieron al análisis de varianza y las medias se compararon con el test de Tukey, a nivel 5\%. Los datos fueron analizados mediante el programa INFOSTAT (2002).

\section{RESULTADOS Y DISCUSIÓN}

\section{Composicion florística}

En los censos realizados se identificaron 31 especies (Tabla 1), de las cuales $58 \%$ corresponde a Gramíneas, $22 \%$ a Leguminosas, $10 \%$ a Ciperáceas y el $10 \%$ restante a otras familias. La mayor parte de las Gramíneas son de ciclo estival (C4) y pertenecen a las tribus Andropogóneas y Paníceas. La presencia de Gramíneas y Leguminosas de ciclo invernal fue escasa, coincidiendo con las observaciones de Pizzio y Royo Pallarés (1994).

Los datos de la Tabla 1 corresponden al último censo realizado al finalizar el ensayo. En T1 se observó una mayor diversidad en la flora, tanto en gramíneas como en leguminosas. En el tratamiento con mayor intensidad de pastoreo aumentó la frecuencia de especies inverno-primaverales como Biza subaristata y Piptochaetium sp. Entre la leguminosas invernales se encontró Trifolium polymorphum cuya presencia no se registró en T0.

La cobertura del canopeo estuvo distribuida de la siguiente manera: Gramíneas entre 65 y 95\%, Ciperáceas 1 a $6 \%$ con un máximo de $30 \%$ (censo de Enero de 2003), Leguminosas entre 1 y $13 \%$ y otras fami-

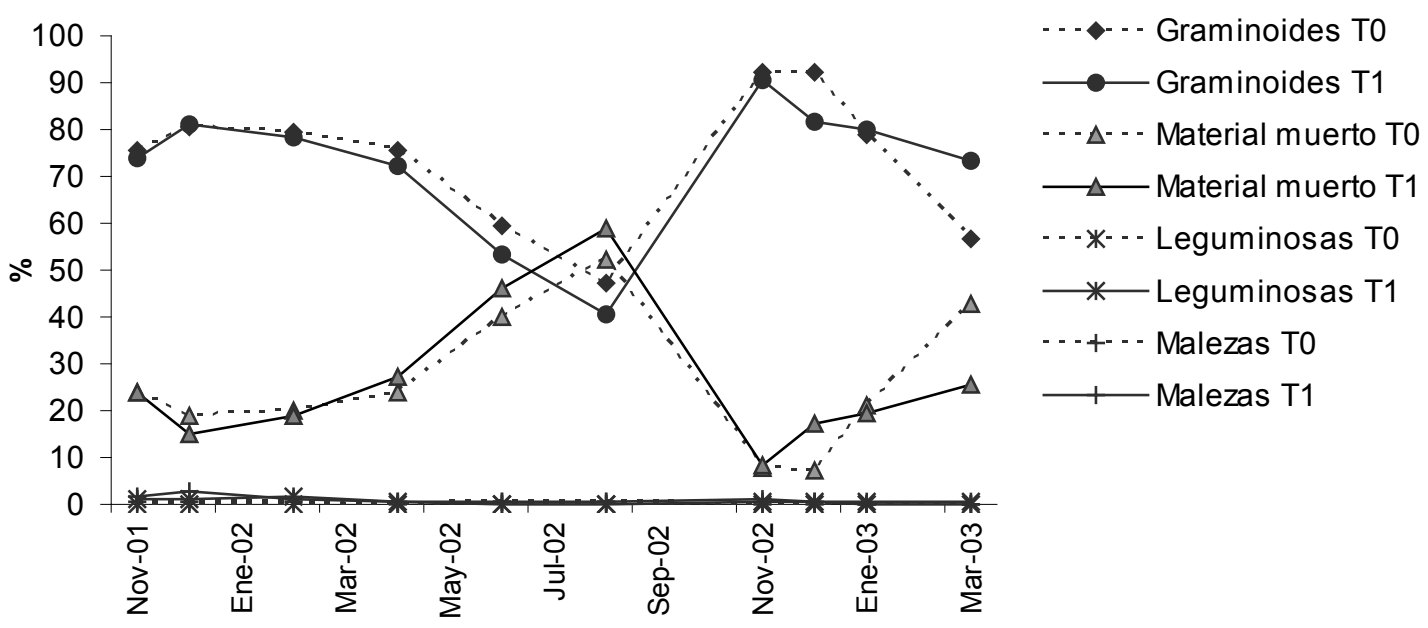

Figura 2 Contribución de los componentes a la producción de MS 
Tabla 1. Componentes vegetales de un pastizal del Departamento Lavalle (Corrientes, Argentina) bajo dos intensidades de pastoreo. $\mathrm{T} 0=$ pastoreo continuo; $\mathrm{T} 1=$ pastoreo rotativo.

\begin{tabular}{|c|c|c|c|c|c|}
\hline \multirow{2}{*}{ Familia } & \multirow{2}{*}{ Tribu } & \multirow{2}{*}{ Especie } & \multirow{2}{*}{ Ciclo (1) } & \multicolumn{2}{|c|}{ Frecuencia (2) } \\
\hline & & & & TO & T1 \\
\hline \multirow{18}{*}{ Gramíneas } & \multirow[t]{5}{*}{ Andropogóneas } & Andropogon lateralis & $\mathrm{P}-\mathrm{e}$ & MA & $\mathrm{MA}$ \\
\hline & & A. selloanus & $\mathrm{P}-\mathrm{e}$ & - & B \\
\hline & & Sorghastrum agrostoides & $\mathrm{P}-\mathrm{e}$ & - & B \\
\hline & & Schizachyrium sp. & $\mathrm{P}-\mathrm{e}$ & - & B \\
\hline & & Rottboellia selloana & $\mathrm{P}-\mathrm{e}$ & - & B \\
\hline & \multirow{8}{*}{ Paníceas } & Paspalum notatum & $\mathrm{P}-\mathrm{e}$ & MA & $A$ \\
\hline & & P. saurae & $\mathrm{P}-\mathrm{e}$ & B & - \\
\hline & & P. plicatulum & $\mathrm{P}-\mathrm{e}$ & B & $\mathrm{M}$ \\
\hline & & Panicum bergii & $\mathrm{P}-\mathrm{e}$ & B & B \\
\hline & & P. milioides & $\mathrm{P}-\mathrm{e}$ & - & B \\
\hline & & Rhynchelitrum sp. & $\mathrm{P}-\mathrm{e}$ & - & B \\
\hline & & Digitaria sanguinalis & $A-e$ & - & B \\
\hline & & Setaria geniculata & $\mathrm{P}-\mathrm{e}$ & - & B \\
\hline & Eragrósteas & Eragrostis lugens & $\mathrm{P}-\mathrm{e}$ & $\mathrm{B}$ & B \\
\hline & Esporobóleas & Sporobolus indicus & $\mathrm{P}-\mathrm{e}$ & $\mathrm{B}$ & - \\
\hline & Festúceas & Briza subaristata & P-ip & - & $\mathrm{B}$ \\
\hline & Agrósteas & Calamagrostis sp. & P-ip & $\mathrm{B}$ & - \\
\hline & \multirow[t]{9}{*}{ Estípeas } & \multirow{9}{*}{$\begin{array}{l}\text { Piptochaetium sp. } \\
\text { Carex sp. } \\
\text { Eliocarix sp. } \\
\text { Cyperus sp. } \\
\text { Stylosanthes sp. } \\
\text { Indigofera asperifolia } \\
\text { Indigofera bongardiana } \\
\text { Desmodium incanum } \\
\text { Chamaecrista sp. } \\
\text { Trifolium polymorphum }\end{array}$} & P-ip & - & B \\
\hline \multirow{4}{*}{ Ciperaceas } & & & $\begin{array}{l}\mathrm{P}-\mathrm{e} \\
\mathrm{P}-\mathrm{e}\end{array}$ & $M$ & $A$ \\
\hline & & & $\mathrm{P}-\mathrm{e}$ & & \\
\hline & & & $\mathrm{P}-\mathrm{e}$ & - & B \\
\hline & & & $\mathrm{P}-\mathrm{pe}$ & $B$ & B \\
\hline \multirow{4}{*}{ Leguminosas } & & & $\mathrm{P}-\mathrm{pe}$ & B & B \\
\hline & & & $\mathrm{P}-\mathrm{pe}$ & $M$ & $A$ \\
\hline & & & $\mathrm{P}-\mathrm{e}$ & - & B \\
\hline & & & $P-i$ & - & B \\
\hline Oxalidáceas & & Oxalis sp. & $\mathrm{P}$ & \multirow{3}{*}{ MA } & \multirow{3}{*}{$\mathrm{MA}$} \\
\hline Umbelíferas & & Hydrocotyle sp. & $\mathrm{P}$ & & \\
\hline Plantagináceas & & Plantago sp. & $P$ & & \\
\hline
\end{tabular}

(1) $\mathrm{P}=$ Perenne; $\mathrm{A}=$ Anual; $\mathrm{e}=$ estival; $\mathrm{i}=$ invernal; $\mathrm{i}=$ inverno-primaveral; $\mathrm{pe}=$ primavero-estival

${ }^{(2)} \mathrm{MA}=$ Muy alta (0,76 a1); $\mathrm{A}=\operatorname{Alta}(0,51$ a 0,75$) ; \mathrm{M}=\operatorname{Media}(0,26$ a 0,5$) ; \mathrm{B}=\mathrm{Baja}(0,25$ ó menos)

lias hasta el 7\%. Estos resultados coinciden con otros autores que estudiaron pastizales de la región (Fernández et al., 1993; Royo Pallarés, 2000; Goldfarb et al., 2000). La especie predominante en el tapiz, con mayor cobertura y frecuencia fue la "paja colorada". Como especie acompañante se destacó el "pasto horqueta" (Paspalum notatum). Entre las leguminosas se destacaron Desmodium incanum (nv: "pega- pega") por ser la especie de mayor cobertura y frecuencia y T. polymorphum (nv: "trébol rosado") por su contribución en el período invernal.

\section{Acumulación de materia seca}

En cuanto a la contribución relativa de los distintos componentes a la MS del pastizal, las Leguminosas y las demás familias de latifoliadas tuvieron una contribución muy escasa. Las primeras registraron un máximo de $1,5 \%$ en $\mathrm{T} 1$, pero en general no alcanzaron el 1\% de la producción total (Figura 2).

El principal aporte estuvo dado por graminiformes y material muerto. La proporción de material verde fue mucho más alta que la de material muerto durante los meses de primavera y verano, aunque se igualaron en el período otoño-invernal. En el corte correspondiente al mes de agosto los valores del componente verde fueron de $47 \%$ y $41 \%$ para $\mathrm{T} 0$ y T1 respectivamente, mientras que el material muerto alcanzó al $52 \%$ y $59 \%$. La mayor acumulación relativa de material muerto fue provocada por la disminución de las temperaturas y las precipitaciones que impidieron el rebrote de la vegetación, y por el ciclo de las especies estivales que alcanzaron la madurez en este período. 
Tabla 2 Producción estacional de MSA $\left(\mathrm{kg} \mathrm{ha}^{-1}\right)$ Departamento Lavalle (Corrientes, Argentina).

\begin{tabular}{l|l|cc|cc|c} 
& \multirow{2}{*}{ Estación } & \multicolumn{2}{|c|}{ T0 } & T1 & CV \\
\hline \multirow{4}{*}{ Año 1 } & Primavera & 2065,75 & $\mathrm{~b}$ & 2688,44 & $\mathrm{a}$ & 12,62 \\
& Verano & 2374,25 & $\mathrm{a}$ & 2882,40 & $\mathrm{a}$ & 14,13 \\
& Otoño & 1142,95 & $\mathrm{a}$ & 1078,63 & $\mathrm{a}$ & 19,61 \\
& Invierno & 354,57 & $\mathrm{~b}$ & 648,2 & $\mathrm{a}$ & 32,57 \\
\cline { 2 - 7 } & Total & 5937,52 & & 7297,67 & \\
\hline \multirow{3}{*}{ Año 2 } & Primavera & 3795,49 & $\mathrm{a}$ & 4198,63 & $\mathrm{a}$ & 15,22 \\
& Verano & 4100,24 & $\mathrm{a}$ & 4815,73 & $\mathrm{a}$ & 10,28 \\
\cline { 2 - 7 } & Total & 7895,73 & & 9014,36 & & \\
\hline
\end{tabular}

Letras distintas en una fila indican diferencias significativas. Tukey $p \leq 5 \%$

En el último corte (Marzo '03) se observaron diferencias entre ambos tratamientos. En T0 se acumuló una mayor cantidad de hojas secas, producto del subpastoreo y la selectividad de los animales propios del sistema de pastoreo continuo, coincidiendo con Damè, et al. (1999). Los remanentes del período estivo-otoñal generalmente son de baja calidad y palatabilidad, con la consiguiente disminución del consumo.

En T1 prevaleció el aporte relativo del componente graminiforme, que estaba constituido principalmente por Andropogon lateralis y Paspalum notatum acompañados por otras especies megatérmicas como, P. plicatulum y Panicum bergii entre otras (Tabla 1).

Asumiendo que los animales consumen solo la biomasa verde (Cid, Brizuela y Kitllein, 2002), solo se analizó la MSA total y la distribución estacional de dicho componente.

La producción de forrajimasa total del primer año fue menor a la del primer semestre del segundo año de evaluación. Durante la primavera y el verano del segundo período coincidieron altas precipitaciones y temperaturas (Figura 1) que determinaron valores de acumulación máximos. La diferencia entre años de este período (primavero-estival) fue aproximadamente $3400 \mathrm{~kg} \mathrm{MS} \mathrm{ha}^{-1}$ en ambos tratamientos (Tabla 2). Esto ratifica la correlación entre producción de forrajimasa y precipitaciones.

El primer año, la producción tuvo una importante fluctuación estacional, registrándose el máximo durante el verano y el mínimo en el invierno. En el primer verano y el otoño no se registraron diferencias significativas entre los tratamientos. En invierno se detectaron diferencias significativas. La MSA en T1 fue significativamente superior y prácticamente duplicó a T0. Una adecuada alternancia entre períodos de pastoreo y descanso, que es la base o el principio de utilización del pastoreo rotativo, permite a las plantas recomponer sus reservas y resistir los períodos desfavorables.

La defoliación intensa que caracteriza al sistema de pastoreo rotativo intensivo, disminuye la selectividad entre especies evitando la acumulación de grandes cantidades de material muerto que inciden desfavorablemente en la capacidad de rebrote.

\section{CONCLUSIONES}

Durante el período de evaluación no se registraron grandes variaciones en la composición botánica. En el tratamiento $\mathrm{T} 1$ hubo una tendencia a incrementar la frecuencia de especies forrajeras invernales.

Con pastoreo rotativo intensivo se produjo una mayor acumulación de forrajimasa durante el período crítico invernal.

De acuerdo a los resultados obtenidos, podría esperarse que, con el pastoreo rotativo intensivo se supere en parte la deficiencia en la oferta forrajera invernal.

\section{BIBLIOGRAFÍA}

Bissio, J.C. 1984. Manejo de pastizales naturales. Conceptos y breve introducción a las técnicas utilizadas. EEA Reconquista INTA, Santa Fe, Argentina. Miscelaneas $\mathrm{N}^{\circ} 2,22 \mathrm{pp}$.

Capurro, R.A. y Escobar, E. 1990. Suelos de la Provincia de Corrientes. En Atlas de Suelos de la República Argentina. SAGyP- INTA- PNUD. Vol I: 517-590.

Carnevali, R. 1994. Fitogeografía de la Provincia de Corrientes. Gobierno de la Provincia de CorrientesINTA: 18-23.

Cid, M.S., Brizuela, M.A. y Kitllein, M. 2002. Beneficio nutricional del pastoreo en manchones en pasturas dominadas por festuca alta. Rev. Arg. Prod. Anim. Vol. 22, Supl. 1: $192-193$.

Fernández, G.F.; Benítez, C.A.; Royo Pallarés, O.; Pizzio, R. 1993. Principales forrajeras nativas del medio este de la provincia de Corrientes. Serie técnica $\mathrm{N}^{\circ}$ 
23. 2a Edición. INTA- EEA Mercedes, Corrientes, Argentina, 91pp .

Fuhlendorf, S.D., Zhang, H., Tunnell, T.R., Engle, D.M.; Cross, A.F. 2002. Effects of grazing on restoration of Southern Mixed Prairie Soils. Restoration Ecology Vol. 10 (2): 401-407

Goldfarb, M.C.; Casco, J.F. y Bernardis, A.C. 2000. Efecto de quemas prescriptas sobre la dinámica y tendencia de un pastizal del NO de Corrientes. I. Indice de tendencia y cobertura. Reunión de Comunicaciones Científicas y Tecnológicas UNNE , 4 pp.

INFOSTAT .2002. Infostat version 1.1- Grupo Infostat, FCA, Universidad Nacional de Córdoba, Argentina

Pizzio, R.M. y Royo Pallarés, O. 1994. Utilización y manejo de los pastizales del ecosistema Campos de
Argentina. IICA-BID-PROCISUR. Dialogo XLUtilización y manejo de pastizales: 115-126

Pizzio, R.M. 1995. Manejo de los campos naturales de la región este de la Provincia de Corrientes. Ganadería Subtropical 95: 67-75.

Roig, F.A. 1977. El cuadro fitosociológico en el estudio de la vegetación. Deserta 4:45-66.

Royo Pallarés, O. 2000. Situación de los pastizales en el ecosistema "Campos" del Mercosur. Situación Actual y potencial Productivo de los Pastizales de Corrientes. $23^{\circ}$ Congreso Argentino de Producción Animal. Suplemento 2: 25-38.

Tomei, C. E. 1995. Manejo racional de los campos naturales para mejorar la producción ganadera en la región chaqueña. Ganadería Subtropical 95: 77-82. 